\title{
Effect of Fluid Supplementation on the Duration of Phototherapy
}

\author{
JESMEEN MORSHED ${ }^{1}$, MOHAMMOD SHAHIDULLAH ${ }^{2}$, MAHBUB MOTANABBI $^{3}$, SANJOY KUMER DEY ${ }^{4}$
}

\begin{abstract}
Background: Hyperbilirubinemia or jaundice is a common condition found in neonates. If intervention is not initiated in case of gradually increasing hyperbilirubinemia may progress to develop kernicterus. Subclinical dehydration (Serum osmolality $>290$ $\mathrm{mosm} / \mathrm{kg}$ ) due to evaporative losses and poor intake of breast milk can lead to an increased incidence and exaggeration of jaundice in newborns leading to longer duration of phototherapy. Adequate hydration and good urine output improve the efficacy of phototherapy.
\end{abstract}

Objective: To evaluate the effect of fluid supplementation on the duration of phototherapy.

Methods: This was a randomized controlled trial conducted in NICU of BSMMU, Dhaka, Bangladesh. Sixty-four term neonates with severe nonhemolytic hyperbilirubinemia (TSB $>18 \mathrm{mg} / \mathrm{dl}$ to $<25 \mathrm{mg} / \mathrm{dl}$ ) was included for study. The subjects were randomized to a case group (Group-I) (intravenous fluid supplementation) and control group (Group-II).

Result: The rate of fall of S. bilirubin per hour at $6 \mathrm{hrs}, 12 \mathrm{hrs}, 24 \mathrm{hrs}$ and $48 \mathrm{hrs}$ after admission in intravenous fluid receiving group was more than the control group and it was statistically significant. Duration of Phototherapy in babies receiving extra IV fluid (Group-I) was also significantly less than those who did not receive IV fluid.

Conclusion: Increased fluid supplementation during treatment in severe non-hemolytic hyperbilirubinemic patient decreases the duration of phototherapy.

Key words: Fluid Supplementation, Hyperbilirubinemia, Neonate, Duration, Phototherapy

\section{Introduction:}

Jaundice is a clinical condition faced in neonatal medicine commonly. About $60 \%$ normal newborn becomes clinically jaundiced during the first week of life. Significantly elevated levels of bilirubin might lead to bilirubin encephalopathy and subsequently kernicterus with devastating, permanent neurodevelopmental handicaps. ${ }^{1}$

Hyperbilirubinemia or jaundice is a common condition found in neonates, especially in Asian races. ${ }^{2}$ The usual peaking of bilirubin occurs at the age of 3 to 5 days but it may continue to rise when there is

1. Medical Officer, Department of Paediatrics, BSMMU, Dhaka

2. Professor of of Neonatology \& Ex. Pro-Vice Chancellor, BSMMU, Dhaka

3. Associate Professor, Department of Paediatrics, BSMMU, Dhaka

4. Associate Professor, Department of Neonatology, BSMMU, Dhaka

Correspondence: Dr. Jesmeen Morshed, Medical Officer, Department of Paediatrics, BSMMU, Dhaka, Mobile: 01711150119 , E-mail: jesm32@gmail.com

Received: 14 April, 2017 impairment of bilirubin elimination like inadequate feeding, infection or prematurity. ${ }^{3}$ Seasonal variation in the incidence of hyperbilirubinemia has been observed, with an increase in summer months. ${ }^{4}$ Subclinical dehydration due to evaporative losses and poor intake of breast milk can lead to an increased incidence and severity of jaundice in newborns. Three fourth of neonates with severe hyperbilirubinemia have subclinical dehydration at presentation (Serum osmolality > $290 \mathrm{mosm} / \mathrm{kg}$ ). ${ }^{5}$ In the extreme neonatal jaundice, progressive reduction in the serum bilirubin levels using phototherapy and exchange transfusion may be necessary. Phototherapy has some side effects like diarrhoea, skin rash, dehydration, overheating, feeding disruption and impairment of mother-baby bonding. During phototherapy in infants, the resultant photoproducts responsible for the decline in serum bilirubin levels are excreted in both urine and bile. Infants also experience increased water loss due to insensible water and stool water loss. 
Fluid supplementation may play role in treating extreme hyperbilirubinemia. Fluid supplementation may result in the decrease in enterohepatic circulation and then a lower rate of bilirubin reabsorption from the bowel. Also, it seems that additional fluid therapy can cause dilution of serum bilirubin and also increases blood circulation in the kidneys and rising urine output and subsequently improves excretion of water soluble photo isomers in urine ${ }^{6}$. High serum bilirubin levels can cause sleepiness in jaundiced newborns. Due to this sleepiness of deeply jaundiced babies, the intake of breast feeding of a newborn's auto regulatory mechanisms may be influenced ${ }^{7}$. Hence, inadequate oral feeding in such sleepy babies, along with increased insensible water loss during phototherapy delays the reduction of serum bilirubin in newborns not receiving extra fluid. However, very little information is available on the effects of providing extra fluids to infants presenting with severe jaundice. This randomized controlled trial was conducted to determine whether fluid supplementation given to term neonates presenting with severe hyperbilirubinaemia can decrease the duration of phototherapy.

\section{Methods:}

This study was a randomized controlled trial carried out in the department of Neonatology, BSMMU, Dhaka, Bangladesh over one year (July 2014 to June 2015). This study was done as a prerequisite of MD examination in paediatrics. IRB (Institutional Review Board) of BSMMU gave ethical clearance for this study.

\section{Inclusion criteria:}

Healthy, breast fed term ( $\geq 37$ weeks gestation) baby Non hemolytic hyperbilirubinemia

Total Serum Bilirubin (TSB) $>18 \mathrm{mg} / \mathrm{dl}[308 \mu \mathrm{mol} / \mathrm{L}]$ to $<25 \mathrm{mg} / \mathrm{dl}$ [ $427 \mu \mathrm{mol} / \mathrm{L}]$

\section{Exclusion criteria :}

Major congenital malformation. (Congenital heart disease, Tracheoesophageal fistula)

Evidence of haemolysis (positive direct Coomb's test, evidence of hemolysis in peripheral blood smear or reticulocyte count is $>6 \%$ )

Clinically dehydrated baby (sunken fontanel, reduced skin turgor, dry mucosa, tachycardia, delayed capillary refill, excessive weight loss) History of giving IV fluid.

Acute bilirubin encephalopathy, Infection (congenital or acquired), Perinatal asphyxia, Infant of diabetic mother.
Study subjects were randomly placed into two groups by using a random number table. Case or intervention group (Group-I) included 30 patients who were given IV fluids $30 \mathrm{ml} / \mathrm{kg}$ besides breast feeding and control group (Group-II) consisted of 30 patients who received only breast milk. Both groups were treated with phototherapy (PT). Phototherapy was introduced by neoBLUE LED phototherapy machine by natus medical incorporated and ARDO Amelux phototherapy lamp (UK). NeoBlue LEDs emit blue light in the 450$470 \mathrm{~nm}$ spectrum. This range corresponds to the peak absorption wave length $(458 \mathrm{~nm})$ at which bilirubin is broken down. By monitoring of newborn from time to time, it was ensured that overhydration or any other complication due to additional iv fluid does not occur. A questionnaire was filled up containing demographic information, case history, examination finding, lab investigation and diagnosis.

Data were stored and analyzed with standard computer software (SPSS-15). Student's t-test and Chi-square test were done to analyze the data according to the nature of data. Probability value of $<0.05$ was considered statistically significant.

\section{Study procedure:}

The term neonates ( $\geq 37$ weeks gestation) with nonhemolytic hyperbilirubinemia were randomly placed into two groups by using a random number table. Group-I was given IV fluid supplementation with $10 \%$ dextrose in $0.225 \%$ saline at a rate of $30 \mathrm{ml}$ per kg per day through peripheral vein after admission to hospital. The infant with mild dehydration (3-5\% of body weight loss) has few clinical signs or symptoms. As beacause our studied group have no clinical signs of dehydration, we decided to give lower range of $(30 \mathrm{ml} /$ $\mathrm{kg}$ ) IV fluid supplementation to the case group. The control group (Group-II) received only breast milk. Both groups received phototherapy. During exposure to light, the eyes were shielded to prevent retinal damage. The position was changed frequently so that maximum skin was exposed to light. Neonates were kept under the light round the clock and taken out only for feeding or changing wet napkins.

The following clinical and laboratory variables were monitored : (1) Total Serum Bilirubin (TSB) at admission and then 6 hours, 12 hours, 24 hours and 48 hours. Total serum bilirubin was measured by SIMENS Dimension RxL Max Integrated Chemistry System. (2) Hydration status by monitoring of body weight, vital signs, urine output. (3) Any clinical signs 
of bilirubin encephalopathy (lethargy, hypotonia, convulsion).

When bilirubin was decreased below phototherapy range according to charts of American Academy of Pediatrics 2004, phototherapy was discontinued.

\section{Results:}

Table-I showing postnatal age on admission in groupI and group-II were not statistically significant. Figure$\mathrm{I}$ is showing that in group-I, $53.3 \%$ male baby and
$46.7 \%$ female baby where as in group-II $60 \%$ male baby and $40 \%$ female baby.

Table-Il showed that the rate of fall of serum bilirubin $(\mathrm{mg} / \mathrm{dl})$ per hour at $6 \mathrm{hrs}, 12 \mathrm{hrs}, 24 \mathrm{hrs}$ and $48 \mathrm{hrs}$ after admission in extra intravenous fluid group is more than the control group (Group-II) and it was statistically significant $(P<0.001)$. Table-III showed that statistically significant $(p<0.001)$ reduction of duration of phototherapy in babies receiving extra intravenous fluid (group-I).

Table-I

Demographic characteristics of the neonates in two groups $(n=60)$

\begin{tabular}{lccc}
\hline Variables & Group-l & Group-II & $\mathrm{p}$ \\
& $(\mathrm{n}=30)$ & $(\mathrm{n}=30)$ & value \\
\hline Post natal age on admission (days) & $3.96 \pm 0.63$ & $4.11 \pm 0.64$ & 0.39 \\
Weight during admission (g) & $2842.7 \pm 596.5$ & $3048.0 \pm 433.1$ & 0.13 \\
\hline
\end{tabular}

Unpaired Student's 't' test was performed to compare between groups.

Group-I= Intervention group

Group-II= Control group

Table-II

Rate of fall of serum bilirubin level ( $\mathrm{mg} / \mathrm{dl})$ per hour in different follow up $(n=60)$

\begin{tabular}{lccc}
\hline Rate of fall of serum bilirubin $(\mathrm{mg} / \mathrm{dl})$ per hour & Group-I $(\mathrm{n}=30)$ & Group-II $(\mathrm{n}=30)$ & p value \\
\hline At 6 hours & $0.55 \pm 0.31$ & $0.27 \pm 0.11$ & $<0.001^{*}$ \\
At 12 hours & $0.45 \pm 0.11$ & $0.33 \pm 0.09$ & $<0.001^{*}$ \\
At 24 hours & $0.30 \pm 0.05$ & $0.24 \pm 0.05$ & $<0.001^{*}$ \\
At 48 hours & $0.18 \pm 0.03$ & $0.16 \pm 0.02$ & $0.001^{*}$ \\
\hline
\end{tabular}

Unpaired Student's 't' test was performed to compare between groups.

$*=$ Significant

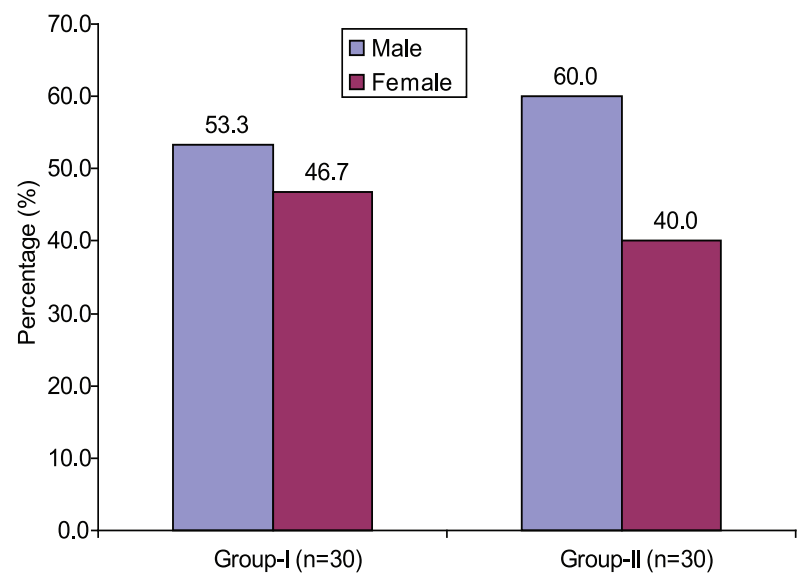

Fig.-1: Gender distribution of the neonates.
Table-III

Duration of phototherapy of two groups $(n=60)$ and outcome.

\begin{tabular}{cccc}
\hline Outcome & $\begin{array}{c}\text { Group-I } \\
(\mathrm{n}=30)\end{array}$ & $\begin{array}{c}\text { Group-II } \\
(\mathrm{n}=30)\end{array}$ & p value \\
\hline \multicolumn{4}{c}{ Duration of phototherapy } \\
$<12$ hrs & $20(66.7 \%)$ & $3(10.0 \%)$ & \\
$12-24$ hrs & $10(33.3 \%)$ & $23(76.7 \%)$ & $<0.001^{*}$ \\
$24-48$ hrs & $(0.0 \%)$ & $4(13.3 \%)$ & \\
\hline
\end{tabular}

Chi-square test (Yates correction) was performed to compare between groups.

* = Significant 


\section{Discussion:}

Postnatal age and weight during admission in both group-I and group-II were not statistically significant $(P=0.39$ and $P=0.13$ respectively) (Table-I). Similar findings were also reported by the study done by Patel et al. ${ }^{7}$ and Punaro et al. ${ }^{8}$

In this study 16 (53.3\%) male baby and 14 (46.7\%) female baby were included in fluid supplemented group, whereas in non fluid supplemented group 18 $(60 \%)$ male baby and $12(40 \%)$ female baby.

In our study, Rate of fall of serum bilirubin ( $\mathrm{mg} / \mathrm{dl}$ ) per hour at $6 \mathrm{hrs}, 12 \mathrm{hrs}, 24 \mathrm{hrs}$ and $48 \mathrm{hrs}$ after admission in extra intravenous fluid group is more than the control group (group II) and it is statistically significant (TableII). Mehta $S$ et al showed TSB levels were similar in the two groups at inclusion but were significantly lower at 4 and 8 hours in the extra fluid receiving group. Which is similar of our study.

In our study, fluid intervention group (Group-I) required phototherapy only up to 24 hours to decrease the bilirubin level below phototherapy range. On the other hand, control group (Group-II) needed upto 48 hours of phototherapy (Table-III). The duration of phototherapy was statistically less in intervention group. This may be due to the fact that fluid supplementation perhaps resulted in decrease in enterohepatic circulation and reduced bilirubin reabsorption leading to shorter duration of phototherapy. Similar types of findings were observed by Barrington and Sankaran ${ }^{10}$. Saeidi et al. ${ }^{9}$ also concluded in their study that additional parenteral fluid therapy to icteric newborn may accelerate serum bilirubin reduction in the first 24 hours.

In this study, no patients needed exchange transfusion. None of the patients developed any serious fluid related complication.

\section{Limitation:}

This was a single center study and sample size was small. Serum osmolality not measured.

\section{Conclusion:}

In this study, we found that additional intravenous fluid to newborn with jaundice during phototherapy helps significantly in reduction of serum bilirubin than nonsupplemented group. It also helps in reducing the duration of phototherapy.

\section{Recommendation:}

If fluid administration is monitored properly, additional intravenous fluid besides breast feeding may help to reduce duration of phototherapy and accelerate the rate of fall of serum bilirubin. So whenever phototherapy is given, additional fluid infusion may be considered for smooth recovery. This is to be further verified by large scale study.

\section{References:}

1. Maisels MJ, McDonagh AF. Phototherapy for Neonatal Jaundice. N Engl J Med. 2008;358: 920-8.

2. Janjindamai $W$, Tansantiwong T. Accuracy of transcutaneous bilirubinometer estimatcs using BiliCheck in Thai Neonates. J Med Assoc Thai. 2005; 88:187-90.

3. Bhat YR and Rad A. Transcutaneous Bilirubin in Predicting Hyperbilirubinemia in term Neonates. Indian Journal of Paediatrics 2008;75:119-23.

4. Mehta S, Kumar P, Narang A. A randomized controlled trial of fluid supplementation in term neonates with severe hyperbilirubinemia. J pediatr 2005;147:781-5.

5. Balasubramanian K, Kumar P, Saini SS, Attri SV, Dutta S. Isotonic versus hypotonic fluid supplementation in term neonates with severe hyperbilirubinemia- a double - blend randomized, controlled trial. Acta Paediatrica 2012;101: 236-41.

6. Porter ML, Dennis BL. Hyperbilirubinemia in the term newborn. Am Fam Physician 2002;65: 599-606.

7. Patel M, Munshi S, Mehariya KM. Effect of Fluid Supplementation in Severe Neonatal Hyperbilirubinemia. International Journal of Science and Research (IJSR) 2014;3: 2524-6.

8. Punaro E, Maria AM, Fernando PF. Systematic follow-up of hyperbilirubinemia in neonates with a gestational age of 35 to 37 weeks. Journal de Pediatria. 2011;87: 301-6.

9. Saeidi R, Farhad H, Vahideh F. Role of intravenous extra fluid therapy in icteric neonates receiving phototherapy. Saudi Med J. 2009; 30:1176-9.

10. Barrington KJ, Sankaran K. Guidelines for detection, management and prevention of hyperbilirubinemia in term and late preterm newborn infants. Paediatr Child Health 2007; 12:1B-12B. 